\title{
Imbalance Prediction Among Elderly People Using Deep Learning
}

\author{
Oussema Fakhfakh ${ }^{1}$, Imen Megdiche ${ }^{1} \Gamma$, Rejane Dalce ${ }^{1}$ and Thierry Val ${ }^{2} \Gamma$ \\ ${ }^{1}$ Institut de Recherche en Informatique de Toulouse, Institut National Universitaire Champollion, ISIS Castres, France \\ ${ }^{2}$ Institut de Recherche en Informatique de Toulouse, Université Toulouse 2 Jean Jaurès, France \\ \{oussema.fakhfakh, imen.megdiche, rejane.dalce, thierry.val\}@irit.fr
}

\begin{abstract}
Keywords: Imbalance Prediction, Sensors, Deep Learning, Strong/Weak Signals, Eldery People
Abstract: As the elderly demographic grows larger, the need for efficient healthcare becomes pressing. One solution is to introduce Artificial Intelligence in the healthcare domain, which implies relevant dataset exploitation. In this paper, we address the issue of imbalance prediction using open-access sensor-based datasets recorded on both young adults and senior citizens. We highlight the need to adapt feature selection according to the population age : strong signals for one population may be classified as weak for another. Although the obtained results are encouraging, there remains a need for adaptive models developed using AI and under the guidance of medical experts.
\end{abstract}

\section{INTRODUCTION}

The growing number of elderly people and particularly in developed countries rises health challenges. As the state of elderly people can rapidly decline from robust to frail to dependant, the need for smart monitoring systems endowed with artificial intelligence becomes pressing.

Developing artificial intelligence based on the data generated from Iot devices is challenging in the case of elderly people studies: (i) first, gathering data is restricted for various reasons (medical, ethical...). So far, we found only one dataset (Sisfall) (Sucerquia et al., 2017) registering falls concerning one elderly person, (ii) second, deep learning or machine learning needs a huge amount of labelled data to train efficient models. If we consider fall or imbalance problems their observation rate for elderly people would not be sufficient. That's why we found datasets (Kessentini et al., 2018)(Ojetola et al., 2015) generated by young participants imitating elderly problems.

Based on these facts, we propose in this paper an experimental study that investigates the cross evaluation of deep learning models generated on datasets observed on elderly and young people. The aim of this study is to highlight alternative methods to develop deep learning approaches combining both young and elderly observations. The topic of our interest is imbalance prediction as it is less addressed in the literature compared to fall and this degradation in the gait occurs before fall.
The remainder of this paper is organized as follows : after reviewing the existing studies on fall and imbalance detection, we introduce our experimental settings, the datasets and the model architecture. We then present the obtained results and conclude the paper with a brief discussion and future work.

\section{RELATED WORK}

Imbalance prediction for elderly people is a topic related to the preservation of autonomy. This topic is much less studied in the literature compared to fall detection. Considering the correlation between imbalance and fall, we present in this section the most relevant studies related to our proposition. We summarize in table 11 related work on either fall or imbalance with a particular focus on the devices used, the data mining algorithm employed and the possible preprocessing techniques.

Concerning fall detection systems, we can distinguish three types of approaches : Camera-based, sensor-based and hybrid approaches. As for camerabased approaches, these solutions generate images data that can be analysed for movement tracking. The principal drawbacks of these solutions are :(i) the restrictive rules to the subject movement, (ii) the need for a well equipped environment along with cameras and dedicated storage. This environment is predominately indoor in elderly people houses. For this category, we cite the work of (Adhikari et al., 2017) which 
uses a Kinect to track subject's movement and implements a convolutional network in order to process captured images and classify human activities.

As for sensor-based approaches, we cite the work of (Casilari et al., 2017) who propose a star topology composed of a smartphone and SensorTags to record motion data. The smartphone acts as a central node and also produces acceleration data. The SensorTags placed on the ankle, waist, right wrist and chest of the subject, generate data through a 3D gyroscope and a 3D magnetometer. The work of (Sucerquia et al., 2017) also includes an accelerometer and a gyroscope mounted in the belt buckle. The collected data are available in the dataset SisFall ${ }^{1}$. The authors of (Musci et al., 2018) used SisFall to classify human activities into three categories : fall, near fall and ADLs using a Long Short Term Memory architecture and achieved an accuracy of $97 \%$. The authors of (Mahfuz et al., 2018) used both SisFall and another open source dataset called 'MobiAct'. They achieved an accuracy of $98.75 \%$ in ADLs and falls classification task using a deep neural network. The authors performed preprocessing on the raw data of SisFall and MobiAct as well as new features generation.

Regarding accessory-mounted sensors, the work of (Huang et al., 2008) uses a smart cane that contains force sensors and a CCD camera. A two-state finite state machine has been implemented containing 'Normal walking mode' and 'Falling down mode'. In this work, the distance between the center of legs and the cane was investigated in order to classify the subject's activity. The work of (Kessentini et al., 2018) presents a system that contains a smart cane connecting to ChisteraPi gateway. In this study, a dataset was created by letting young adult subjects experience some ADLs and falls. They developed a KNearest Neighbors classifier achieving 97\% accuracy.

Hybrid approaches mix data obtained by cameras and sensors. The work presented by (Kwolek and Kepski, 2014) uses a wearable device mounted near the pelvis, containing a 3D accelerometer and a 3D gyroscope, and a smart Kinect camera. Data generated are used in a threshold based algorithm to detect fall then compared with the results of an SVM model (99.67\% of accuracy).

To conclude this section, we noticed that papers focusing on fall detection consider activities like Stumbling or Near Fall, besides Forward Fall, backward Fall and lateral Fall while others consider these activities as ADLs. Compared to these works, we will focus on the non-fall state and investigate the ADLs related to imbalance (or as said near fall) states in or-

1 http://sistemic.udea.edu.co/en/ investigacion/proyectos/english-falls/
Table 1: Summary on related work

\begin{tabular}{|c|c|c|c|}
\hline Approach & Connected device & Algorithm & Preprocessing \\
\hline Chen et al., 2015) & $\begin{array}{l}\text { Smartphone enabled } \\
\text { 3D Accelerometer }\end{array}$ & $\begin{array}{l}\text { Finite state } \\
\text { machine }\end{array}$ & No \\
\hline Roeing et al., 2017) & $\begin{array}{l}\text { Cell phone that } \\
\text { contains invasive } \\
\text { sensors }\end{array}$ & not reported & No \\
\hline Mahfuz et al., 2018 & Smartphone & $\begin{array}{l}\text { Deep neural } \\
\text { network }\end{array}$ & Yes \\
\hline Fontecha et al., 2013) & 3D accelerometer & Affinity Tree & Yes \\
\hline Musci et al., 2018) & $\begin{array}{l}\text { 3D accelerometer/ } \\
\text { 3D gyroscope }\end{array}$ & $\begin{array}{l}\text { Long Short } \\
\text { Term Memory }\end{array}$ & No \\
\hline Sucerquia et al., 2017) & $\begin{array}{l}\text { 2*3D accelerometer/ } \\
\text { 3D gyroscope }\end{array}$ & $\begin{array}{l}\text { Threshhold based } \\
\text { classification } \\
\text { algorithm }\end{array}$ & Yes \\
\hline Huang et al., 2008, & Smart Cane & $\begin{array}{l}\text { Finite State } \\
\text { machine }\end{array}$ & No \\
\hline Casilari et al., 2017) & $\begin{array}{l}\text { 3D accelerometer/ } \\
\text { 3D gyroscope/ } \\
\text { 3D magnetometer }\end{array}$ & not reported & No \\
\hline Kwolek and Kepski, 2014 & $\begin{array}{l}\text { 3D accelerometer/ } \\
\text { 3D gyroscope }\end{array}$ & $\begin{array}{l}\text { Support Vector } \\
\text { machine }\end{array}$ & Yes \\
\hline Kessentini et al., 2018; & $\begin{array}{l}\text { Cane/Teensy 3.2/ } \\
\text { Lora Receiver }\end{array}$ & $\begin{array}{l}\text { K-Nearest } \\
\text { Neighbors }\end{array}$ & No \\
\hline
\end{tabular}

der to establish predictive models and study their relevance.

\section{DEEP LEARNING FOR IMBALANCE PREDICTION}

In this section we present the datasets used, the data preprocessing performed on them and the architecture of the ANN network applied on our study.

\subsection{Datasets}

We identified two datasets for imbalance prediction. These datasets, Sisfall (Sucerquia et al., 2017) and Cogentlab (Ojetola et al., 2015), record falls and ADL activities.

Table 2 compares both datasets. Sisfall is observed on eldery people, while Cogentlab is observed on young people so that we can study the stability of imbalance model, the relevance of features as well as the cross evaluation between models. Table 3 lists the features in each dataset with their explanations. Given these common criteria, it is easy to compare results of these datasets.

Table 2: Sisfall vs Cogentlab comparison

\begin{tabular}{|c|c|c|}
\hline & Cogentlab & SisFall \\
\hline Sampling rate & $100 \mathrm{~Hz}$ & $200 \mathrm{~Hz}$ \\
\hline Subjects & $\begin{array}{l}42 \text { healthy adults } \\
-6 \text { female } \\
-36 \text { male }\end{array}$ & $\begin{array}{l}38 \text { participants } \\
-15 \text { elderly } \\
-23 \text { young adult } \\
\text { only one elderly person recordings }\end{array}$ \\
\hline Activities & $\begin{array}{l}-14 \text { falls } \\
- \text { ADLs for } 23 \text { minutes }\end{array}$ & ADLs and Fall activities \\
\hline Sensors & $\begin{array}{l}\text { Shimmer sensor } \\
\text { - 3D accelerometer } \\
\text { - 3D gyroscope }\end{array}$ & $\begin{array}{l}-2 * 3 \mathrm{D} \text { accelerometer } \\
-3 \mathrm{D} \text { gyroscope }\end{array}$ \\
\hline Axis orientation & $\begin{array}{l}-\mathrm{x} \text {-axis : orthogonal } \\
\text { to the walking direction } \\
\text { - y-axis : upwards } \\
\text { - } \mathrm{z} \text {-axis : forward direction }\end{array}$ & $\begin{array}{l}\text { - } x \text {-axis : right side of the } \\
\text { participant } \\
\text { - y-axis : gravity direction } \\
\text { - z-axis forward direction }\end{array}$ \\
\hline Algorithm & J48 Decision Tree & Thresh-hold based algorithm \\
\hline Accuracy & $94.4 \%$ & not reported \\
\hline
\end{tabular}


Table 3: Features description

\begin{tabular}{|l|l|}
\hline Feature & Description \\
\hline $\mathrm{Lx}$ & Linear acceleration in the $\mathrm{x}$ direction (orthogonal ) \\
\hline $\mathrm{Ly}$ & Linear acceleration in the y direction ( upwards) \\
\hline $\mathrm{Lz}$ & Linear acceleration in the $\mathrm{z}$ direction ( Forward) \\
\hline $\mathrm{Gx}$ & Angular Velocity or Rotation in the $\mathrm{x}$ direction ( orthogonal) \\
\hline $\mathrm{Gy}$ & Angular Velocity or Rotation in the y direction ( upwards) \\
\hline $\mathrm{Gz}$ & Angular Velocity or Rotation in the $\mathrm{z}$ direction ( Forward) \\
\hline Roll & Rotation around the front-to-back axis \\
\hline Pitch & Rotation around the side-to-side axis \\
\hline Yaw & Rotation around the vertical axis \\
\hline
\end{tabular}

\subsection{Data Preprocessing}

In order to prepare our datasets for deep learning tasks, we perform these preprocessing steps :

- Features computation;

- Target column extraction to obtain a binary classification problem (Imbalance / Not Imbalance);

- Feature scaling to get normalized data.

\subsubsection{Features Computation}

We note that for the Sisfall the acceleration and rotation features are originally in bits. To convert them into gravity, as suggested by the authors of Sisfal ${ }^{2}$ we use the following equations:

$$
\begin{aligned}
\text { Acceleration }[g] & : \frac{2 * \text { Range }}{2^{\text {Resolution }}} * \text { AccelBin } \\
\text { AngularVelocity }\left[{ }^{\circ} / s\right]: & \frac{2 * \text { Range }}{2^{\text {Resolution }}} * \text { RotationBin }
\end{aligned}
$$

For both SisFall and Cogentlab datasets, we computed the orientation data : pitch, roll, yaw using he formulas cited in (Lachtar et al., 2019).

\subsubsection{Target Extraction}

CogentLab For imbalance state, we took the records corresponding to the Near-fall activity ("loss imbalance" label) and extract rows annotated by the normal walking. The resulting dataset for our study contains 313503 rows including 211866 corresponding to the normal walking state and 101637 corresponding to the imbalance case.

Sisfall We took data that corresponds to the label (Stumbling while walking) recorded for the 60-year old subject. We extracted also rows corresponding to the label (normal walking) recorded by the same subject. The resulting dataset for our study contains 31999 rows including 19999 corresponding to the normal walking state and 12000 corresponding to the imbalance case.

\footnotetext{
2 http://sistemic.udea.edu.co/wp-content/ uploads/2015/11/Readme1.txt
}

\subsubsection{Feature Scaling}

For our datasets, we investigate two types of scaling features standardization and Min-Max scaling. For standardization we used this formula :

$$
z=\frac{x-v}{s}
$$

Where $\mathrm{z}$ represents the modified value, $\mathrm{v}$ indicates the variance of the feature $\mathrm{x}$ and $\mathrm{s}$ designates its standard deviation.

For Min-Max scaling, we used this formula :

$$
X \text { norm }=\frac{X-X \min }{X \max -X \min }
$$

Where Xnorm represents the obtained value after performing scaling, Xmin indicates the minimum value of the feature $\mathrm{X}$ and $\mathrm{Xmax}$ designates its maximum value.

Experimentations show that standardization is more suitable and efficient than Min-Max scaling based on the accuracy obtained in these two cases.

\subsection{The ANN Model Architecture}

In this paper, we implemented a fully connected neural network based on following architecture:

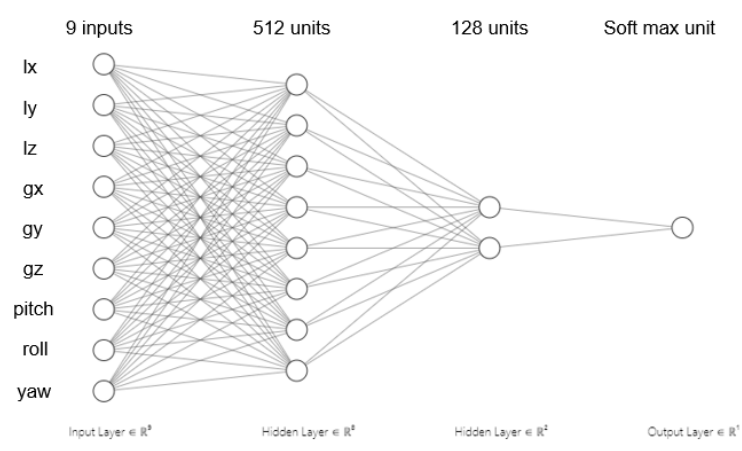

Figure 1: ANN architecture

- The input layer has 9 input units corresponding to the features of Table 3

- The first hidden layer contains 512 neurons and uses the ReLu activation function,

- The second hidden layer contains 128 neurons and also uses the ReLu activation function,

- The output layer for the binary classification uses the softmax activation function.

The choice of the different tuning parameters is done heuristically. First, we have investigated both the ReLu and Tanh functions for the hidden layers. The model with ReLu gave better results than the 
one which used Tanh in particular with the Cogentlab dataset. This dataset being the largest, we retain the ReLu function.

Concerning the number of neurons (units) which are generally a power of 2 , we tried all possible combinations in the interval $[32-512]$. Simulations show that 512 for the first hidden layer and 128 for the second one give the best results.

Finally, for the final activation function used in the output layer, we experiment both sigmoid and softmax for our binary classification problem. Simulations showed that Softmax is more precise than sigmoid in term of correct predictions.

\section{EVALUATION RESULTS}

The aim of our evaluations is twofold: (1) check the relevance of deep learning approaches for predicting imbalance based on sensor's data , (2) demonstrate experimentally that there is a subtlety between weak signals and strong signals for the prediction of imbalance that should be considered when constructing deep learning models. The first objective is presented in the subsection 4.1. The second objective is addressed in subsections 4.2 and 4.3

We note that the evaluation results presented in this section were produced on a computer with an Intel core i5 CPU and a 8 GB memory. The code source was developed on the Anaconda platform with the python 3.7 version. Several libraries were employed such as numpy, pandas, matplotlib, seaborn, tqdm, sklearn for data preparation, keras for deep learning and eli 5 for feature importance determination.

\subsection{Model Evaluation}

Based on the architecture presented in previous section, we carried out two evaluations on SisFall and CogentLab data sets. These evaluations followed the same experimental settings : (i) 100 epochs, (ii) 64 batch size, (iii) the Adam implementation for the gradient descent algorithm, (iv) the categorical crossentropy for the loss function and (v) 33\% for test set , $67 \%$ for train test.

As shown in Figure 2, we can observe the evolution of the Accuracy both on the train (green and blue curves) and test (orange and red curves) sets through the 100 epochs. The accuracy of our model applied on these datasets for the train exceeds $90 \%$ which is a very interesting result. We can notice, for the Cogentlab dataset, that the model starts overfitting on the train set after the 30 th epoch. This can be explained by the volume of this dataset compared to the Sisfall dataset. But we highlight that we have a stability on the test set so this overfitting problem can be fixed by a good tuning of the number of epochs. In this example, 40 epochs are sufficient to get a stable and good model for both datasets.

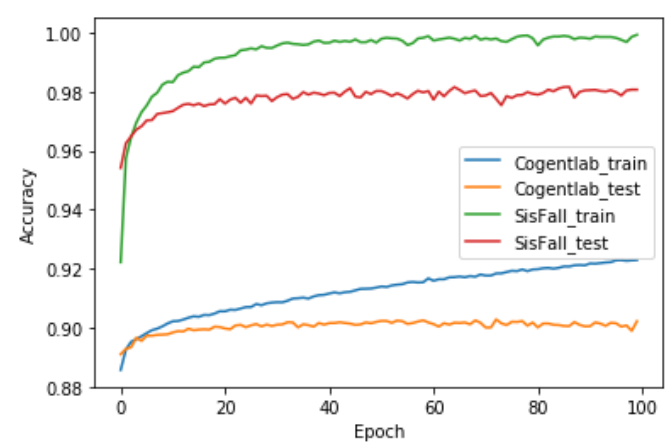

Figure 2: Model accuracy evolution through 100 epochs

\subsection{Feature Importance Determination}

Discovering the most important characteristic(s) in our study will lead to determining the most suitable sensors for elderly people monitoring. To evaluate the feature importance, we use the elis 5 library. The concept is to eliminate one feature at a time, run the training process and compute the accuracy of the model. The most significant feature is the one which absence most degrades the accuracy score. A second complementary alternative consists in observing the weights of each feature according to the model by running it with all the features.

Features observations for Cogentlab and Sisfall Regarding the Cogentlab dataset, Figure 3 summarizes the importance and the weights of the different features. These figures show that the angular velocity features generated by the 3D gyroscope, along the $x$ and $\mathrm{z}$ axes, and the pitch are the most influential features in imbalance prediction. These observations are similarly coherent with the work of (Lachtar et al., 2019). Regarding Sisfall, we see in Figure 4 that the most important features are the linear acceleration data obtained by the $3 \mathrm{D}$ accelerometer.

Discussion. We observe a significant difference in the prioritization of the features between Cogentlab and Sisall. Therefore, we can make the following assumption : elderly people movement is affected the most by the linear acceleration while young adult

3 https://eli5.readthedocs.io/en/latest/ overview.html 


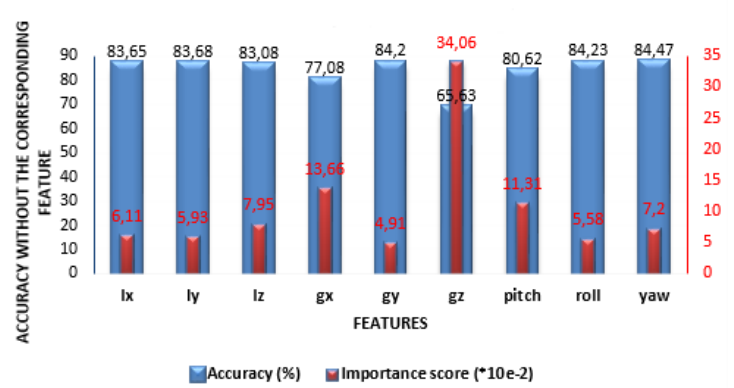

Figure 3: Features importance for Cogentlab

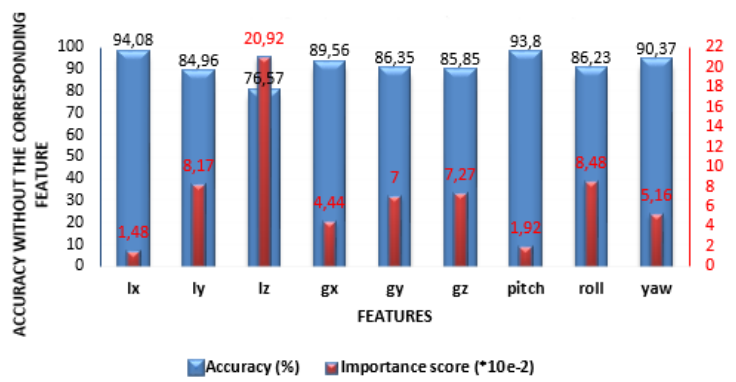

Figure 4: Features importance for SisFall

movement is influenced the most by the angular velocity. This means that the observation of imbalance with sensors gives off two types of signals :

- The strong signals are the most important features for the elderly people using sensors. These signals are close to the state of fall or in advanced stages of imbalance,

- The weak signals are the most influential features for the young adult movement which are in the same time among the least significant features for the elderly people movement. These signals can be observed for the starting stages of the imbalance problems.

So for elderly people with advanced state imbalance, the prediction models will be efficient with strong signals. But for eldery people on the starting stages of imbalance or in the middle stages, we should think on new strategies of constructing the prediction models.

\subsection{A Cross Evaluation Of Models}

In this section, we study the impact of weak and strong signals on the quality of predictions on both datasets. We proceed by a cross evaluation of models. We use three settings: (1) Setting 1 consists in applying the prepared ANN on each complete data set separately, then saving each model and applying a cross evaluation of models, (2) Setting 2 consists in using only weak signals as features (which are in our case rotation and pitch data) and applying a cross evaluation of models, (3) Setting 3 consists in using only strong signals as features (which are in our case acceleration and pitch data) and applying a cross evaluation of models. For all these settings, let model1 refer to the ANN trained on SisFall, while model 2 refers to the ANN trained on Cogentlab.

Setting 1 observations As shown in Table 4 when we evaluate Cogentlab using modell we get an accuracy of $47 \%$, whereas, the application of model 2 on Sisfall gives an accuracy of 57\%. We have an important decrease compared to the application of each model on its dataset. Therefore, it's clear that our models with both weak and strong signals aren't stable. These results suggest that a composite process of evaluation taking advantage of both signals separately would be better.

Table 4: Setting 1 results

\begin{tabular}{|l|c|c|}
\cline { 2 - 3 } \multicolumn{1}{c|}{} & SisFall Model & Cogentlab Model \\
\hline SisFall & $98 \%$ & $57 \%$ \\
\hline Cogentlab & $47 \%$ & $90 \%$ \\
\hline
\end{tabular}

Setting 2 observations As shown in Table 5, when applying model 1 in SisFall using only weak signals, we get an accuracy of $84 \%$. We note a decrease in accuracy from $98 \%$ to $84 \%$ by using weak signals. In contrast, evaluating model 2 in Cogentlab data set using only weak signals gives us an accuracy of $88 \%$. When applying modell in Cogentlab we get an accuracy of $60 \%$, while the evaluation of SisFall using model 2 gives us $72 \%$. These results confirm the relevance of weak signals in the imbalance detection when observed individually.

Table 5: Setting 2 results

\begin{tabular}{|l|c|c|}
\cline { 2 - 3 } \multicolumn{1}{c|}{} & SisFall Model & Cogentlab Model \\
\hline SisFall & $84 \%$ & $72 \%$ \\
\hline Cogentlab & $60 \%$ & $88 \%$ \\
\hline
\end{tabular}

Setting 3 observations As shown in Table 6 for Cogentlab data set, we evaluated it using model 2 and we get an accuracy of $76 \%$. The decrease from $90 \%$ to $76 \%$ can be explained by the absence of the most significant features in the prediction of imbalance for the young adult subjects present in Cogentlab. For the model stability verification in this context, we applied modell in Cogentlab which gave us a score of 
$51 \%$ while trying model 2 in SisFall returned a score of $33 \%$.

Table 6: Setting 3 results

\begin{tabular}{|l|c|c|}
\cline { 2 - 3 } \multicolumn{1}{c|}{} & SisFall Model & Cogentlab Model \\
\hline SisFall & $89 \%$ & $33 \%$ \\
\hline Cogentlab & $51 \%$ & $76 \%$ \\
\hline
\end{tabular}

We observe from these results a potential complementarity in prediction between the weak signals model, applied on young adult subjects and the strong signals models, applied on elderly subjects. The results suggest that a twofold model would perform better in this case: by following a composite process, the model based on the weak signals would produce correct predictions in situations where the strong signals model would fail and vice versa.

\section{CONCLUSION}

In this paper we presented an experimental study to showcase new hypothesis related to imbalance prediction for elderly populations. Thanks to an artificial neural network applied on Congentlab and SisFall datasets and a cross evaluations of models, we observed a complementarity between weak and strong signals. Our results are encouraging but should be validated by medical experts and tested on larger populations. For future work, we develop new strategy of deep learning compositions in the light of weak and strong signals. It will be validated on a new collected scalable dataset based on a smart stick. The Iot device is connected with three communication technologies LoRa, BLE and WiFi. We plan to collect data for both young and eldery peoples on nursing residences.

\section{REFERENCES}

Adhikari, K., Bouchachia, H., and Nait-Charif, H. (2017). Activity recognition for indoor fall detection using convolutional neural network. In 2017 Fifteenth IAPR International Conference on Machine Vision Applications (MVA), pages 81-84.

Casilari, E., Santoyo-Ramón, J. A., and Cano-García, J. M. (2017). Umafall: A multisensor dataset for the research on automatic fall detection. Procedia Computer Science, 110:32-39.

Chen, P.-H., Li, Y.-H., Chiou, C. W., Lee, C.-Y., and Lin, J.-M. (2015). A smart safety cane for human fall detection. International Journal of Ad Hoc and Ubiquitous Computing, 20(1):49-65.
Fontecha, J., Navarro, F. J., Hervás, R., and Bravo, J. (2013). Elderly frailty detection by using accelerometer-enabled smartphones and clinical information records. Personal and ubiquitous computing, 17(6):1073-1083.

Huang, J., Di, P., Wakita, K., Fukuda, T., and Sekiyama, K. (2008). Study of fall detection using intelligent cane based on sensor fusion. In 2008 International Symposium on MicroNanoMechatronics and Human Science, pages 495-500. IEEE.

Kessentini, O., Dalce, R., Megdiche, I., and Bastide, R. (2018). Towards predicting frailty symptoms through a smart walking stick. In 2018 IFIP/IEEE International Conference on Performance Evaluation and Modeling in Wired and Wireless Networks (PEMWN), pages 1-7.

Kwolek, B. and Kepski, M. (2014). Human fall detection on embedded platform using depth maps and wireless accelerometer. Computer methods and programs in biomedicine, 117(3):489-501.

Lachtar, A., Val, T., and Kachouri, A. (2019). Elderly monitoring system in a smart city environment using LoRa and MQTT. IET Wireless Sensor Systems, 2043-6386:(on line).

Mahfuz, S., Isah, H., Zulkernine, F., and Nicholls, P. (2018). Detecting irregular patterns in iot streaming data for fall detection. In 2018 IEEE 9th Annual Information Technology, Electronics and Mobile Communication Conference (IEMCON), pages 588-594. IEEE.

Musci, M., De Martini, D., Blago, N., Facchinetti, T., and Piastra, M. (2018). Online fall detection using recurrent neural networks. arXiv:1804.04976.

Ojetola, O., Gaura, E., and Brusey, J. (2015). Data set for fall events and daily activities from inertial sensors.

Roeing, K. L., Hsieh, K. L., and Sosnoff, J. J. (2017). A systematic review of balance and fall risk assessments with mobile phone technology. Archives of gerontology and geriatrics, 73:222226.

Sucerquia, A., López, J. D., and Vargas-Bonilla, J. F. (2017). Sisfall: A fall and movement dataset. Sensors, 17(1):198. 